\title{
Learning to live with early stage dementia involved a continuous process of adjustment that comprised 5 stages
}

Werezak L, Stewart N. Learning to live with early dementia. Can J Nurs Res 2002;34:67-85.

\section{QUESTION: How do older adults learn to live with early stage dementia?}

\author{
Design \\ Grounded theory.
}

\section{Setting}

Saskatchewan, Canada.

\section{Participants}

3 women and 3 men (age range 61-79 y) with a diagnosis of Alzheimer's disease or related disorder and early stage dementia. All participants lived at home with their spouses.

Source of funding

not stated.

For correspondence: Ms L Werezak,

Saskatchewan Institute of Applied Science and Technology, Kelsey Campus, Saskatoon, Saskatchewan, Canada. werezak@siast.sk.ca

\section{Methods}

Each person participated in 2 semistructured interviews. Transcripts from the first interviews were analysed using constant comparison, and 5 preliminary categories were identified. During second interviews (1-3 mo later) the author validated and expanded on these 5 categories.

\section{COMMENTARY}

The use of interview data in research with people with dementia is limited by several factors, including reliability of data, appropriate staging of disease, and ethical issues of consent. However, studies from the UK and US have shown that people living with dementia have perspectives and concerns that are different in focus and importance from those of family caregivers, ${ }^{1-3}$ with whom most dementia research is done.

Werezak and Stewart did a grounded theory study with 6 older adults, producing a structured framework that describes 5 stages in adjusting to early stage dementia. Although the model was developed from interviews with a small homogeneous sample (all participants were well educated, married, and financially secure), it will undoubtedly be recognised by practitioners, given its findings that adjustment to dementia is a continuous process influenced at each stage by the person's developing and ever changing level of awareness.

The study by Werezak and Stewart has important implications for clinicians working in the field, in particular the need for discussion, support, and consideration of how the diagnosis is communicated, given the effects on those with early stage dementia. Support groups were identified as being especially helpful, although the fact that 4 of the 6 participants were attending support groups may have influenced this finding.

From this modest study, hypotheses can be generated for use in designing and testing interventions and support strategies for people living with early stage dementia. Additional qualitative studies can also be designed to explore the relevance of the study's grounded theory with groups that are different from the homogeneous sample used in Werezak and Stewart's study. The study's findings can sensitise healthcare providers to the potential effects of their interactions on people with early stage dementia. Sue Hall, RMN, MSc Senior Lecturer, School of Health and Social Care Oxford Brookes University John Radcliffe Hospital Oxford, UK

1 Keady J, Nolan M. Younger onset dementia: developing a longitudinal model as the basis for a research agenda and as a guide to interventions with sufferers and carers.J Adv Nurs 1994:19:659-69.

2 Killick J, Allan K. Communication and care of people with dementia. Buckingham: University Killick J, All

Sabat SR. Voices of Alzheimer's disease sufferers: a call for treatment based on personhood. J Clin Ethics 1998;9:35-48.

\section{Main findings}

A theoretical framework outlined the continuous process of adjusting to early stage dementia, which comprised 5 stages: antecedents, anticipation, appearance, assimilation, and acceptance. Different levels of awareness connected the stages. As participants moved through the stages, their awareness shifted from highly introspective to more outwardly focussed.

(1) Antecedents included subprocesses that made it difficult to obtain a diagnosis of dementia. Recognising that a problem exists: most participants were initially unaware of their memory problems, largely because of the insidious onset of symptoms, which they attributed to benign forgetfulness or work stress. When discussing the onset of memory loss, many participants recalled their previous memory quality, identified family members with and without dementia, and often described confounding health problems that made it difficult for them to identify which disease process was causing their memory loss. (2) Anticipation. Participants anticipated obtaining a diagnosis and described common reactions of shock and horror. They then began considering future losses, anticipating losing the ability to care for themselves and worrying about "becoming a burden". Participants felt anxious about telling others about their memory loss, wondering how people would respond. (3) Appearance. Participants slowly became aware that family, friends, and co-workers were noticing their memory loss. They recounted hurtful situations where others were insensitive or indifferent to their disease related experiences. Participants described seeing themselves as the same person, despite their memory impairments. (4) Assimilation referred to the cyclical and continuous process of fitting dementia into one's life. Participants described assimilating the disease into their inner world (eg, feelings and thoughts about the disease, physiological changes) and their outer world (eg, lifestyle changes related to memory loss, interacting with supportive and unsupportive significant others). Positive mediating experiences, such as feeling supported by others and retaining abilities that gave life meaning and purpose, facilitated the transition from inner to outer worlds. In response to negative mediating experiences, such as disease progression and inability to function in social or work situations, participants withdrew into their inner world. (5) Acceptance. All participants described achieving a degree of acceptance of their dementia that allowed them to focus on enjoying the remainder of their lives. Factors facilitating such acceptance included others' acceptance of the disease, use of humour, hope, and altruism.

\section{Conclusion}

People with early stage dementia engaged in a continuous process of adjusting that comprised 5 stages, each linked by differing levels of awareness: antecedents, anticipation, appearance, assimilation, and acceptance. 